\title{
The SERS Activity of a Supported Ag Nanocube Strongly Depends on Its Orientation Relative to Laser Polarization
}

\author{
Joseph M. McLellan, Zhi-Yuan $\mathrm{Li}^{\dagger}$, Andrew Siekkinen, and Younan Xia* \\ Department of Chemistry, University of Washington, Seattle, Washington 98195-1700 \\ ${ }^{\dagger}$ Institute of Physics, Chinese Academy of Sciences, Beijing 100080, P. R. China \\ *Corresponding author. E-mail: xia@chem.washington.edu
}

\section{Experimental Procedures}

Synthesis of Silver Nanocubes. In a typical synthesis, $5 \mathrm{~mL}$ of ethylene glycol (EG, J. T. Baker) was placed in a $20-\mathrm{mL}$ vial, capped, and heated with magnetic stirring in an oil bath at $150{ }^{\circ} \mathrm{C}$ for $1 \mathrm{~h}$. $0.75 \mathrm{~mL}$ of $12 \mathrm{mM} \mathrm{HCl}$ in EG was then quickly added into the vial, and the vial was recapped. After $10 \mathrm{~min}, 1.5 \mathrm{~mL}$ each of $94 \mathrm{mM} \mathrm{AgNO}_{3}$ (Aldrich) and $147 \mathrm{mM}$ poly(vinyl pyrrolidone) (PVP, M.W.=55,000, Aldrich, the concentration was calculated in terms of the repeating unit), both dissolved in EG, were simultaneously added through a two-channel syringe pump (KDS-200, KD Scientific, Holliston, MA) at a rate of $22.5 \mathrm{~mL}$ per hour into the vial. The vial was then capped and continued with heating at $150{ }^{\circ} \mathrm{C}$ until the solution turned into an ocher color. Upon injection of the $\mathrm{AgNO}_{3}$ solution, the reaction mixture went through a series of color changes that included milky white, light yellow, transparent, red, and ocher. The final product, silver nanocubes with sharp corners, was obtained via centrifugation (30 min at 3,900 rpm) and washed with acetone once and ethanol twice to remove excess EG and PVP and finally redispersed in deionized water for storage. 
Truncation of Silver Nanocubes. To induce truncation, $1 \mathrm{~mL}$ of an aqueous suspension $(\sim 0.5$ $\mathrm{nM}$ ) of the as-synthesized nanocubes was transferred into $1.0 \mathrm{~mL}$ of ethanol and then to $4.5 \mathrm{~mL}$ of EG containing $~ 0.1 \mathrm{mM}$ PVP by centrifugation (5 min at 14,000 rpm) and re-dispersion. The suspension was heated to $145^{\circ} \mathrm{C}$ in a glass vial under magnetic stirring. After $2 \mathrm{~min}, 36.5 \% \mathrm{HCl}$ was added to a total concentration of $1 \mathrm{mM}$, and the mixture was heated for an additional 5 min. Once cooled down to room temperature, the product was centrifuged (30 min at 3,900 rpm) and washed with acetone and then ethanol several times to remove EG and excess PVP, and finally re-dispersed in deionized water.

\section{Instrumentation}

The SEM (or TEM) samples were prepared by placing a drop of the final product (suspended in water) on a silicon wafer (or carbon-coated copper grid), and drying under ambient conditions. SEM images were taken using a Sirion XL field-emission microscope (FEI, Hillsboro, OR) operated at an acceleration voltage of $10 \mathrm{kV}$. TEM imaging was performed using a Philips 420 microscope operated at $120 \mathrm{kV}$. The UV-Vis spectra were obtained with a Hewlett Packard 8452A Diode Array UV-Vis spectrophotometer (Hewlett Packard Corp., Palo Alto, CA). Confocal Raman spectra were obtained with Reninshaw InVia Raman Spectrometer coupled to a Leica DM/LM microscope. The backscattered Raman signals were collected on a CCD detector thermoelectrically cooled to $-60{ }^{\circ} \mathrm{C}$. All spectra were obtained through a 50x objective (NA = 0.80 ) using a $514 \mathrm{~nm}$ argon laser ( $\sim \mathrm{mW}$ at the sample), a holographic notch filter to reject the laser light, a grating with 1800 lines per millimeter, in 1 acquisition, and 30-sec accumulations. The spot size was approximated at $\sim 800 \mathrm{~nm}$ using the expression for a focused Gaussian beam (equation [1]), which is valid when a laser light is focused by a microscope objective and the beam diameter is much smaller than the back diameter of the objective.

$$
\omega_{0}=\frac{4 \cdot \lambda}{\pi \cdot N A}
$$

In this expression $\omega_{0}$ is the minimum waist diameter for a laser beam of wavelength $\lambda$, focused by an objective with a numerical aperture NA. The particles were located in dark-field mode, and optical micrographs were recorded with a Canon A620 digital camera, with a 7.2 megapixel CCD detector. 

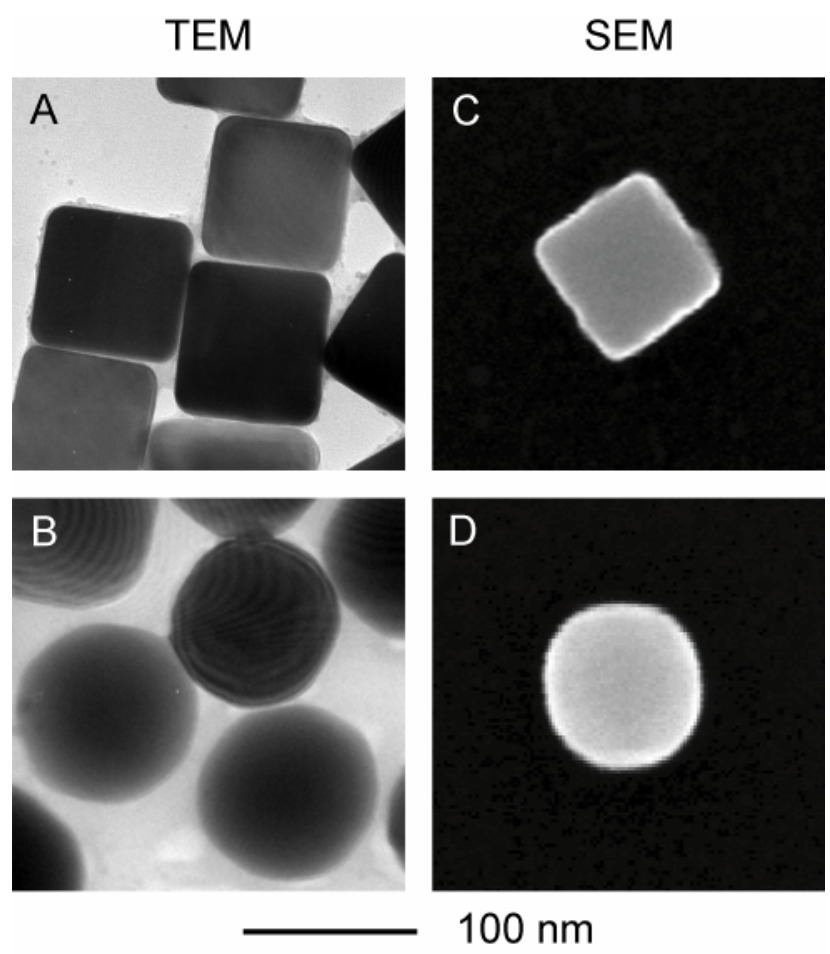

Figure S1. A comparison of typical TEM images (A and B) and SEM images (C and D) of sharp and truncated Ag nanocubes used for correlated SERS and SEM experiments. 


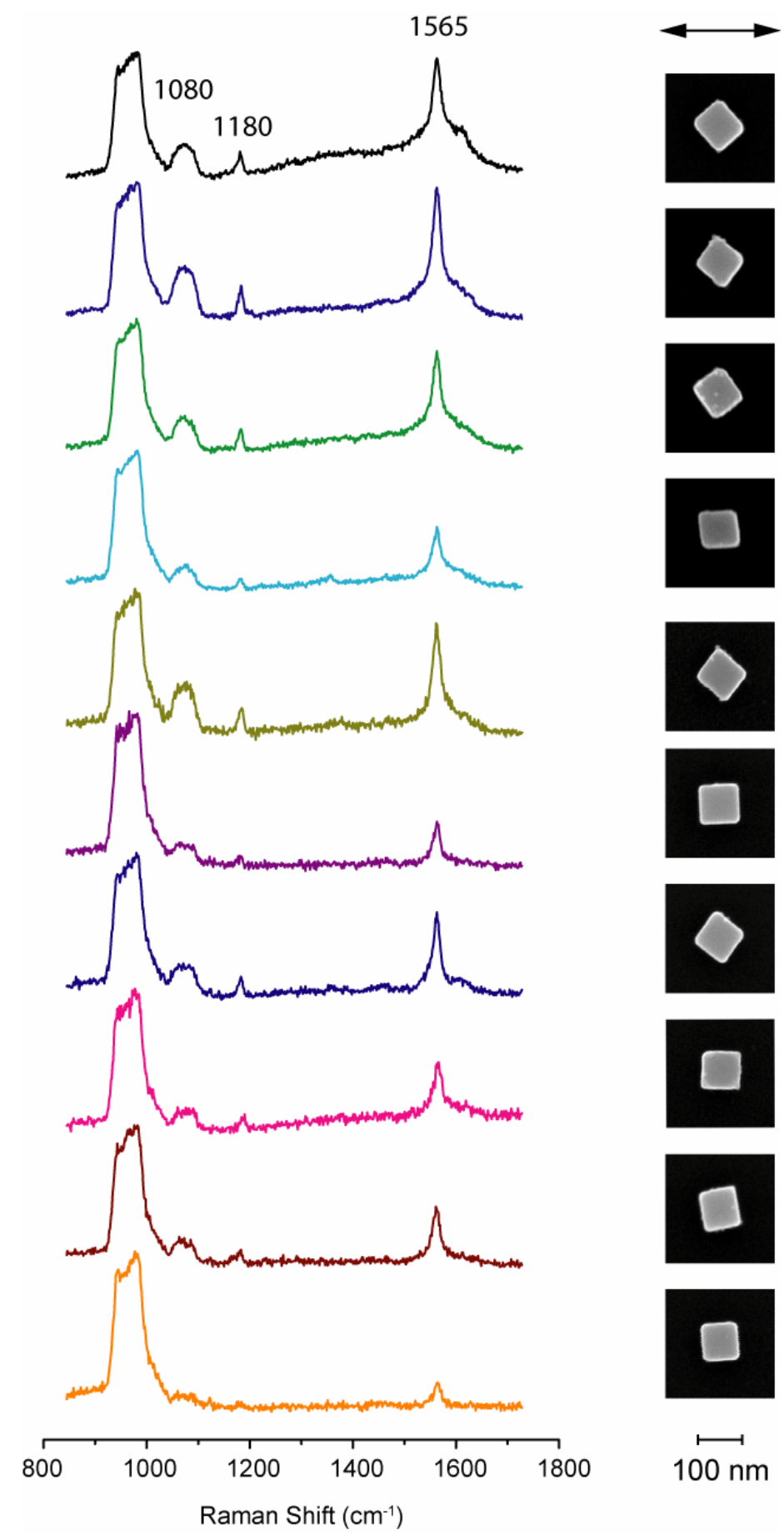

Figure S2. SEM images and the corresponding SERS spectra of 1,4-BDT adsorbed on ten different Ag nanocubes. The images show the morphology and orientation of the cubes, while the black arrow above the images denotes the orientation of the laser polarization. The 100-nm scale bar applies to all the SEM images. Spectra were normalized against the Si peak and stacked with off-set for clarity. 


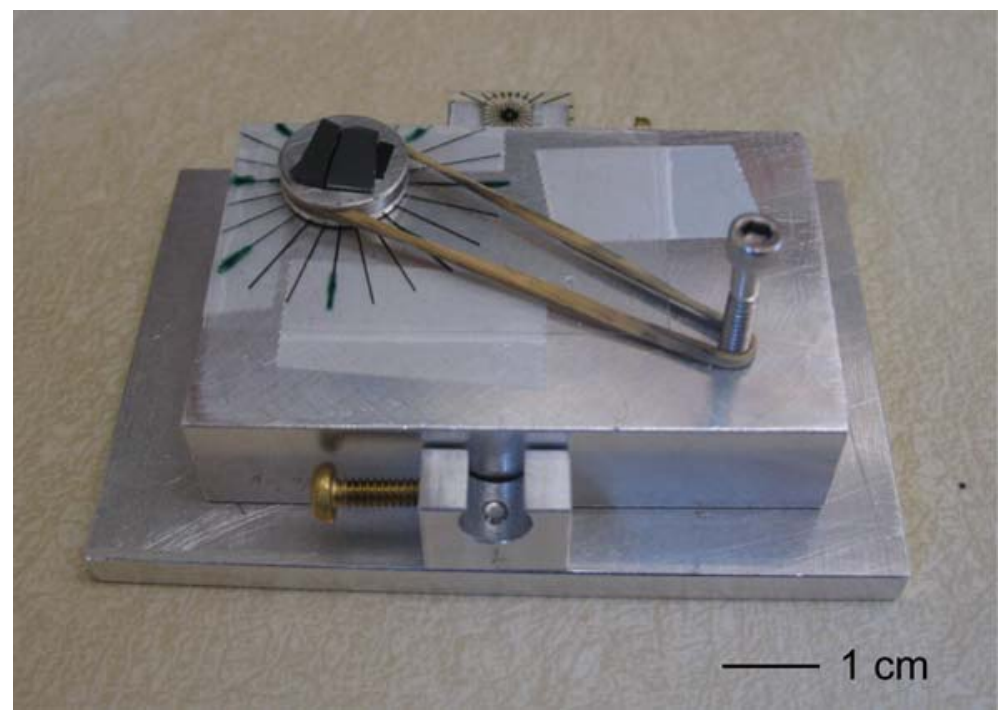

Figure S3. Photograph of a homemade manual rotating stage used for angle-resolved SERS measurements. 